Noordman, J., Dulmen, S. van. The consequences of task delegation for the process of care: female patients seem to benefit more. Women \& Health: 2016, 56(2), 194-207

\begin{tabular}{|l|l|}
\hline $\begin{array}{l}\text { Postprint } \\
\text { Version }\end{array}$ & 1.0 \\
\hline $\begin{array}{l}\text { Journal website } \\
\text { Pubmed link }\end{array}$ & $\underline{\text { http://dx.doi.org/10.1080/03630242.2015.1086467 }}$ \\
\hline DOI & $10.1080 / 03630242.2015 .1086467$ \\
\hline
\end{tabular}

This is a NIVEL certified Post Print, more info at http://www.nivel.eu

\title{
The consequences of task delegation for the process of care: Female patients seem to benefit more
}

\author{
JANNEKE NOORDMAN PHD\& SANDRA VAN DULMEN PHD
}

\begin{abstract}
The shift of tasks from primary care physicians to practice nurses and the continuing incease in the numbers of women involved in medical care may have consequences for the provision of health care and communication. The aim of the present study was to examine potential differences in female practice nurses' application of communication skills, practice guidelines, and motivational interviewing skills during consultations with female and male patients. Nineteen female practice nurses and their patients $(n=181)$ agreed to have their consultations videotaped (during 2010-2011). The videotaped consultations were rated using two validated instruments: the Maas-Global (to assess generic communication skills and practice guidelines) and the Behaviour Change Counselling Index (to assess motivational interviewing skills). Multilevel linear and logistic regression analyses were performed. Female practice nurses provided significantly more comprehensive information during consultations with female patients $(\mathrm{p}=.03)$ and talked more about management with male patients $(\mathrm{p}=.04)$. Furthermore, nurses applied motivational interviewing skills more clearly during consultations with female than with male patients $(\mathrm{p}<.01)$. The shift in tasks from primary care physicians toward practice nurses may have implications for clinical and patient outcomes as patients will no longer be counseled by male professionals. Conceivably, female patients are motivated more by nurses to change their behavior, while male patients receive more concrete management information or advice.
\end{abstract}

\section{INTRODUCTION}

Currently, practice nurses frequently work alongside physicians in general practices all over the world (Bourgueil, Marek, and Mousques 2005; Fairman et al. 2011; Noordman et al. 2013a). This enables physicians to delegate tasks to nurses regarding the counseling of patients with chronic illnesses and their lifestyle behavior (Laurant et al. 2005). In the 5-year period from 2003 to 2008, Dutch patients with a chronic illness substantially increased their visits to their practice nurse from $4 \%$ in 2003 to 
Noordman, J., Dulmen, S. van. The consequences of task delegation for the process of care: female patients seem to benefit more. Women \& Health: 2016, 56(2), 194-207

$39 \%$ in 2008 , while the total number of general practice visits remained stable (Heijmans, Spreeuwenberg, and Rijken2010).

The shift of tasks from primary care physicians toward practice nurses and the continuing increases in numbers of women involved in medical care may have consequences for the provision of health care and communication. As few men enter the nursing profession (Bartfay et al. 2010; Meadus 2000), patients mainly encounter female practice nurses, while among primary care physicians the gender distribution is more equal (i.e., $40 \%$ female physicians in the Netherlands (Hingstman and Kenens 2010)).

A person's gender can affect the communication with a health-care provider (Bertakis, Franks, and Epstein, 2009; Hall and Roter 2002; Street 2002; Van den Brink-Muinen et al. 2002). However, most studies of communication have been conducted only with primary care physicians. These studies for example, have found that, compared to male patients, female patients encountered more patient-centered communication when visiting their primary care physician (Bertakis, Franks, and Epstein,2009; Hall and Roter 2002). However, one older study found that male patients were likely to get more attention from their physician (Meeuwesen, Schaap, and Van der Staak 1991) and another study found that male patients received less biomedical information from their female physician compared to female patients (Street 2002). Furthermore, a study among nurses and elderly patients revealed that patient's gender played only a minor role in the way nurses communicated (CarisVerhallen et al. 1999). In addition, gender concordant visits appeared in one study to last longer than gender discordant visits, especially when these visits involved female physicians and female patients (Franks and Bertakis 2003). The extent to which these gender differences also exist in practice nurse-patient encounters is, as yet, unknown.

Practice nurses' encounters are usually structured around practice guidelines for, e.g., type 2 diabetes, cardiovascular disease prevention, and stopping smoking (Heiligers et al. 2012). Furthermore, motivational interviewing, a patient-centered counseling style for behavior change, is part of Dutch practice nurses' everyday communication and a key element of the practice nurse training (Heiligers et al. 2012). Motivational interviewing focuses on increasing intrinsic motivation to change behavior by helping patients to explore and to resolve ambivalence between desired behavior and actual behavior (Miller and Rollnick 2002). In The Netherlands, practice nurses cannot diagnose patients and are not allowed to refer patients or prescribe medicines without the permission of a primary care physician. However, they do manage consultations independently (Heiligers et al. 2012). Practice nurses working in several of the United States have a comparable level of autonomy, although in many U.S. states, practice nurses are permitted to diagnose patients or prescribe medicines (Fairman et al. 2011).

The aim of the present study was to examine potential differences in female practice nurses' application of communication skills, practice guidelines, and motivational interviewing skills during consultations with female and male patients. Based on previous findings from studies in primary care (Roter, Hall, and Aoki 2002; Van den Brink-Muinen et al. 2002), female practice nurses were expected to communicate more empathically with their female patients compared to their male patients. Furthermore, it was expected that motivational interviewing skills of practice nurses would also be more visible during consultations with female patients because of the 
Noordman, J., Dulmen, S. van. The consequences of task delegation for the process of care: female patients seem to benefit more. Women \& Health: 2016, 56(2), 194-207

patient-centered principles behind motivational interviewing. No differences were expected in practice nurses' application of practice guidelines and discussing lifestyle behaviors of the patient during consultations between male and female patients, as a previous study found no relationship between lifestyle communication provided by nurses and patients' gender (Caris-Verhallen et al. 1999).

\section{METHODS}

\section{Data collection and participants}

Data were derived from a study of communication between practice nurses and patients (Noordman et al. 2012, 2013a,2013b). Our aim was to include twenty practice nurses and we had no exclusion criteria. General practitioners who participated in an earlier study (Noordman et al. 2010) (except for one) were contacted for participation of their practice nurses in the study. Almost half, 47\%, of the practices responded; six practices did not employ a practice nurse. Ten practice nurses from seven practices volunteered to take part. General practitioners from one other practice (health-care center) contacted us for participation of all of their practice nurses $(n=10)$; all nurses volunteered to take part. One practice nurse stopped working during our study and has therefore been omitted. Nineteen practice nurses from eight general practices were included and agreed to have consecutive, everyday consultations videotaped. Approximately ten consultations per nurse were recorded on video. Data collection took place between June 2010 and February 2011. All nurses were trained in motivational interviewing or behavior change counseling as part of their nursing education. Patients were consecutively approached by a researcher in the waiting room, without any exclusion criteria, during one or two random days, and signed a written informed consent form. All adult patients scheduled for an appointment with the practice nurse were eligible for inclusion (90\% agreed to participate, no dropouts).

\section{Ethical considerations}

Our research complied with the Helsinki Declaration and was carried out according to Dutch privacy legislation. The privacy regulations were approved by the Dutch Data Protection Authority. According to Dutch legislation, approval by a medical ethics committee was not required for this observational study. All nurses and patients participating in the study provided written, signed informed consent.

\section{Observations}

The videotaped consultations were coded by two observers independently, using two validated communication assessment instruments: (1) the Maas-Global (Van Thiel, Ram, and Van Dalen 2000) and (2) the Behaviour Change Counselling Index (BECCI; Lane 2002; Lane et al. 2005). The Maas-Global was used to rate nurses' generic communication and clinical competence (i.e., adherence to practice guidelines). This instrument was divided into three sections: communication skills for each separate consultation phase (from introduction until evaluation of consultation), general communication skills (e.g., exploration, information giving, empathy), and clinical aspects (adherence to practice guidelines). Each item has to be rated on a scale ranging from 0 (not present) to 6 (excellent). To assess nurses' adherence to practice guidelines for every consultation, the appropriate guideline was 
Noordman, J., Dulmen, S. van. The consequences of task delegation for the process of care: female patients seem to benefit more. Women \& Health: 2016, 56(2), 194-207

checked; i.e., Dutch College of General Practitioners guidelines on diabetes type 2, cardiovascular disease prevention, chronic obstructive pulmonary disease (COPD), and asthma or the stop smoking guideline (NHG 2012). Some items (e.g., follow-up consultation in case of a first encounter) could be scored as "not applicable" and were left out of the analyses. As practice nurses cannot diagnose patients, the item "diagnosis" of the Maas-Global refers to the diagnosis set by the physician and discussed by the practice nurse during the consultation.

The BECCI was used to assess nurses' application of motivational interviewing skills for every consultation. The BECCI was developed for brief consultations in the health-care setting. This instrument contains eleven, 5-point Likert-scaled items related to the practitioners' behavior and motivational interviewing techniques, ranging from "not at all" to "a great extent." These items are subdivided into four domains: agenda setting and permission seeking (two items), the why and how of change in behavior (five items), the whole consultation (three items), and talk about targets (one item). As recommended by the author of this instrument (Lane 2002), not applicable items were replaced by mean substitution.

Finally, we assessed whether lifestyle behaviors of the patient about smoking, alcohol, physical activity, or nutrition were discussed during the consultation (both on initiative of the practice nurse and the patient). Observer software (Noldus et al.2000) was used to code the video-recorded consultations. The observers were trained researchers with social science backgrounds.

To establish inter-rater reliability, $12 \%$ of the total consultations were coded by both observers independently. Inter-rater agreement was sufficiently high, with average Kappa scores (Sim and Wright 2005) of 0.82 (range 0.54-1.00) for the Maas-Global, 0.81 (range $0.72-1.00$ ) for the BECCI, and 0.91 (range 0.74-1.00) for the discussion about lifestyle behaviors of patients.

\section{Data analysis}

First, characteristics of the practice nurses and the patients were described.

Differences between male and female patients were tested using an independent ttest for continuous variables (i.e., age, consultation duration) and chi-square test for categorical variables (i.e., race/ethnicity, marital status, education level, and [first] disease/complaint). Confounding variables were identified based on significant differences found in practice nurses' and patients' characteristics. The nested-data structure and the likelihood-ratio test revealed the necessity to perform multilevel regression analyses. Therefore, multilevel linear and logistic regression analyses were performed. Two levels were used: patients (level 1) nested within practice nurses (level 2). A null model for every dependent variable (i.e., BECCI domain, Maas-Global item, or lifestyle item) was created. Next, confounding variables (e.g., patient's education level) were added to control for differences in male and female patients (Model 1). Multilevel linear regression was used to determine the association between the four domain scores of the BECCI mean sum score and (almost all) Maas-Global items (dependent variables) with patient's gender (Model 0 and 1). The Maas-Global items, "Introduction," "Request for help," "Evaluation of consultation," and "Emotions," and also the lifestyle items were coded as dichotomous variables (e.g., $0=$ no smoking discussed, $1=$ smoking discussed) and analyzed with multilevel logistic regression (Model 0 and 1), using generalized linear latent and mixed models (gllamm) and the likelihood ratio to assess model fit (Rabe- 
Noordman, J., Dulmen, S. van. The consequences of task delegation for the process of care: female patients seem to benefit more. Women \& Health: 2016, 56(2), 194-207

Hesketh, Skrondal, and Pickles 2002). Male patients were used as reference group. All analyses were performed in State (ver. 12, College Station, TX).

\section{RESULTS}

\section{Characteristics of practice nurses and patients}

In total, 181 consultations between 19 nurses and 181 patients were analyzed. All nineteen practice nurses were female, with a mean age of 42 years (range: $33-$ 52 years) and 4.5 years of experience on average. Eighteen practice nurses reported higher vocational training (i.e., college) as their educational background and one nurse reported lower vocational training.

Fifty percent of the patients were male $(n=90)$; patients were on average 62.6 years of age (range: 18-86 years); 69\% were married or lived together; 79\% were of Dutch ethnic origin (all patients were Dutch citizens); and 51\% of patients stated "vocational training" as their highest educational level. Nearly half of patients, $48 \%$, suffered from type 2 diabetes $(\mathrm{n}=87)$, and 11 had impaired glucose intolerance. Moreover, fifty-two patients had hypertension and twenty-three had asthma or COPD.

No significant differences were observed between male and female patients with respect to age $(p=.89)$, marital status $(p=.27)$, ethnic heritage $(p=.21)$, and (first) disease/complaint $(\mathrm{p}=.22)$. However, male patients in our study had a significantly higher educational level $(\mathrm{p}<.01)$. The consultation duration was on average 22.3 minutes for male patients and 22.7 minutes for female patients visiting the practice nurse $(\mathrm{p}=.77)$.

\section{Generic communication skills of nurses}

The regression coefficients of practice nurses' generic communication skills, according to the Maas-Global during consultations with male versus female patients were controlled for patient's educational level (Table 1). Two significant differences emerged: female practice nurses provided significantly more understandable information (e.g., concrete explanations, understandable language) during consultations with female patients $(\mathrm{p}=.03)$ and talked more about management (e.g., discussing alternatives, risks, and benefits, and determining who will do what and when) with male patients $(\mathrm{p}=.04)$. Female nurses also showed significantly more empathy during consultations with female patients $(\mathrm{p}=.03$; result not shown). However, after controlling for patient's educational level, this difference was no longer statistically significant (Table 1).

\section{[TABLE 1]}

\section{Clinical competence of nurses}

The regression coefficients of nurses' adherence to practice guidelines during consultations with male (reference) versus female patients revealed no significant differences (Table 2). 
Noordman, J., Dulmen, S. van. The consequences of task delegation for the process of care: female patients seem to benefit more. Women \& Health: 2016, 56(2), 194-207

\section{[TABLE 2]}

\section{Motivational interviewing skills of nurses}

Multilevel analyses showed three significant differences regarding nurses' application of motivational interviewing skills during consultations with male patients compared to female patients (Table 3). Overall, female nurses applied motivational interviewing skills more clearly during consultations with female patients ( $p<.01$ for BECCI mean sum score). More specifically, female nurses appeared to pay significantly more attention to "the why and how of change in behaviour" ( $p=.02$ for domain 2$)$ and "the whole consultation" $(p<.01$ for domain 3 ) during consultations with female than with male patients. Furthermore, a trend for "talking about targets" ( $p=.06$ for domain 4$)$ pointed in the same direction. However, regression coefficients ranged from 0.15 to 0.34 , indicating small differences between consultations with male or female patients.

\section{[TABLE 3]}

\section{Discussing patient's lifestyle behavior}

No significant gender differences were found in whether patient's lifestyle behavior was discussed during nurse-patient consultations regarding smoking, alcohol, physical activity, or nutrition (Table 4).

\section{[TABLE 4]}

\section{DISCUSSION}

In the present study, potential gender differences in female practice nurses' application of communication skills, practice guidelines, and motivational interviewing skills during consultations with female and male patients were examined. Several statistically significant associations emerged in the behavior of female practice nurses toward their male versus female patients. First, with respect to general communication skills, this study showed that female practice nurses provided significantly more understandable information (e.g., concrete explanations, understandable language) during consultations with female patients and talked more about management (e.g., discussing alternatives, risks and benefits, and determining who will do what and when) with male patients. Previous studies have shown mixed results. Some relatively old studies have demonstrated that female patients received more comprehensible information than male patients (Hooper et al. 1982; Waitzkin 1985). In contrast, another more recent study found no gender difference in information given to patients by physicians (Hall et al. 1994). Also, Caris-Verhallen et al. (1999) found that nurses in home care generally communicated in the same way with their patients, irrespective of patient's gender. However, patients' gender was also associated with communication differences in some studies. Female patients tend to ask for and provide more information than male patients (Hall and Roter 2002; Hall et al. 1994). Furthermore, another study found that male patients described their health conditions mainly with physical explanations, while female patients used emotional explanations (Hedegaard et al. 2014). This may well be reflected in the communication behavior of nurses, as nurses are likely to be influenced by how their patients behave toward them. Moreover, one study found that the contribution of patients and physicians during consultations was almost equal 
Noordman, J., Dulmen, S. van. The consequences of task delegation for the process of care: female patients seem to benefit more. Women \& Health: 2016, 56(2), 194-207

when they were both female (Hall et al. 1994). We are aware of no prior studies that examined gender differences in the discussion of patient's management (of a disease, treatment, or lifestyle behavior).

Second, the results of the present study suggested, in agreement with our expectations, that female practice nurses applied motivational interviewing skills more clearly during consultations with female than with male patients. To our knowledge, this is the first study that has examined potential gender differences in female practice nurses' application of motivational interviewing skills toward male versus female patients. We expected gender differences based on previous findings, taking into account that motivational interviewing is a patient-centered communication style. As mentioned before, studies have found that female primary care providers (i.e., physicians) and female patients engage in more patient-centered communication than their male equivalents (Bertakis, Franks, and Epstein, 2009; Hall and Roter 2002; Roter, Hall, and Aoki2002; Roter and Hall 2004).

No significant gender differences were found in nurses' adherence to practice guidelines, consultation length, and discussion of patient's lifestyle behaviors with respect to smoking, alcohol, physical activity, or nutrition. This is contrary to the findings of an earlier study that demonstrated that gender concordant visits were longer than gender discordant visits (Franks and Bertakis 2003). However, in agreement with our results, a previous study found no relationship between the lifestyle communication provided by nurses, and patients' gender (Caris-Verhallen et al. 1999). Furthermore, in contrast to our expectation and to preceding findings (Roter, Hall, and Aoki 2002; Van den Brink-Muinen et al. 2002), the female practice nurses in the present study did not communicate more empathically with their female patients compared to their male patients. More explicitly, the significant finding on "empathy" (i.e., female nurses showing more empathy during visits with female patients) was attributed to gender differences in patients' educational level. Overall, the gender differences observed were modest. As pointed out earlier by Street (2002), among others, this is understandable given that gender is one of numerous factors (e.g., age, education) that influence the communicative behavior, beliefs, and perceptions. However, it is important that providers are sensitive to potential differences in patient's gender, especially given the present outcomes on motivational interviewing. The shift in tasks from primary care physicians toward practice nurses may have implications for clinical and patient outcomes as patients will no longer be counselled by male professionals. Male patients can expect more concrete management (e.g., discussing alternatives, risks and benefits, and determining who will do what and when) and less patient-centered communication (i.e., motivational interviewing). Previous research has found that the majority of patients prefer a patient-centered communication style (Swenson et al.2004). Several studies have found that patient-centered communication incorporated into nurses' practice is associated with improved nurse and patient satisfaction, increased adherence to treatment plans, improved patient health, quality of life, and physiological status (Charlton et al. 2008; Lein and Wills 2007), although studies examining the effects of patient-centered communication on patient outcomes are still limited and the (mainly positive) outcomes are often small (Epstein et al. 2005; Stewart et al. 2000).

Furthermore, it is possible that the gender differences we found were a response of the practice nurse to the different needs of female and male patients. For example, 
Noordman, J., Dulmen, S. van. The consequences of task delegation for the process of care: female patients seem to benefit more. Women \& Health: 2016, 56(2), 194-207

male patients could be more resistant to motivational interviewing than female patients. As a result, practice nurses may have provided less patient-centered communication to male patients, but at the same time fulfilled their needs. Moreover, a previous survey study found that the gender of the health-care provider (i.e., physician) or patient in primary care had no influence on patient's satisfaction with their care provider and care (Wolosin and Gesell 2006).

\section{Strengths and limitations}

To our knowledge, this is the first study examining potential gender differences in female practice nurses' application of communication skills, practice guidelines, and motivational interviewing skills during consultations. It is expected that the number of (chronically ill) patients visiting a practice nurse will increase in the near future, which points out the importance of this study. Also, we believe that this is the first study that has observed actual encounters between practice nurses and patients to explore gender differences. Observations are a more objective source than selfreporting by nurses or patients, which could be biased. Another strength was that practice nurses did not know that our study focused on potential gender differences. This study also had some limitations. First, patient's communication behavior was not observed. This could have influenced our results. For example, male and female patients might differ in their initiative to talk about behavior change. However, this seems unlikely because we found no significant gender difference in patient's intention to change behavior (Noordman et al.2013b). Furthermore, no male practice nurses participated in our study so that we were unable to assess gender differences of practice nurses in their communications with patients. However, this does reflect the actual situation in daily practice, as few practice nurses are male (i.e., estimated at less than $3 \%$ by the Dutch association for practice nurses [NVVPO], November 2013). Nonetheless, our findings may not be generalizable because of the limited number of practices and practice nurses that were included and assessed. Also, the Maas-Global was developed to assess the communication skills of physicians. Therefore, some items of the Maas-Global seemed less applicable for practice nurse consultations (e.g., diagnosis). Additionally, one item ("history taking") of the MaasGlobal was not coded reliable (kappa of 0.54). Thus, the outcome and interpretation of results for this item should be treated with great caution. Moreover, confidence in the observations is relatively low because of multiple testing (especially for the Maas-Global items), so that the results of these analyses would need to be replicated. The findings with respect to motivational interviewing (MI) skills are more compelling as they are in a consistent direction and generally stronger. Also, the observers were not masked to patient's gender, which could have contributed to bias in assessment of the communications. In addition, this was a small-scale, exploratory study. Future, larger studies are necessary to replicate our findings. Further studies should examine if and how gender differences in both nurse providers and patients influence patient behavior and health outcomes. Finally, previous literature has been mainly based on gender differences in the physician-patient setting in primary care. Gender differences in physician-patient communication cannot simply be compared to gender differences in nurse-patient communication within primary care. This also applies for comparing nurses in home care to nurses in primary care. Therefore, more studies that investigate potential gender differences in the nursing setting in primary care are essential. 
Noordman, J., Dulmen, S. van. The consequences of task delegation for the process of care: female patients seem to benefit more. Women \& Health: 2016, 56(2), 194-207

\section{ACKNOWLEDGMENTS}

We would like to thank Inge van der Lee for her help in data acquisition and coding of the video-recorded consultations.

\section{FUNDING}

This study was funded by the Dutch Ministry of Health, Welfare and Sport. They had no role in the collection, analysis, interpretation, writing, or decision to submit this article.

\section{REFERENCES}

1. Bartfay, W. J., E. Bartfay, K. A. Clow, and T. Wu. 2010. Attitudes and perceptions towards men in nursing education. The Internet Journal of Allied Health Sciences and Practice 8:17.

2. Bertakis, K. D., P. Franks, and R. M. Epstein. 2009. Patient-centered communication in primary care: Physician and patient gender and gender concordance. Journal of Women's Health 18:539-45. doi:10.1089/jwh.2008.0969.

3. Bourgueil, Y., A. Marek, and J. Mousques. 2005. The participation of nurses in primary care in six European countries, Ontario and Quebec. Issues in Health Economics (IRDES) 95:1-12.

4. Caris-Verhallen, W. M. C. M., I. M. de Gruijter, A. Kerkstra, and J. M. Bensing. 1999. Factors related to nurse communication with elderly people. Journal of Advanced Nursing 30:1106-17. doi:10.1046/j.1365-2648.1999.01197.x.

5. Charlton, C. R., K. S. Dearing, J. A. Berry, and M. J. Johnson. 2008. Nurse practitioners' communication styles and their impact on patient outcomes: An integrated literature review. Journal of the American Academy of Nurse Practitioners 20:382-88. ,

6. Epstein, R. M., P. Franks, K. Fiscella, C. G. Shields, S. C. Meldrum, R. L. Kravitz, and P. R. Duberstein. 2005. Measuring patient-centered communication in patient-physician consultations: Theoretical and practical issues. Social Science \& Medicine61:1516-28. doi:10.1016/j.socscimed.2005.02.001.

7. Fairman, J. A., J. W. Rowe, S. Hassmiller, and D. E. Shalala. 2011. Broadening the scope of nursing practice. The New England Journal of Medicine 364:193-96. doi:10.1056/NEJMp1012121.

8. Franks, P., and K. D. Bertakis. 2003. Physician gender, patient gender, and primary care. Journal of Women's Health 12:73-80. doi:10.1089/154099903321154167.

9. Hall, J. A., J. T. Irish, D. L. Roter, C. M. Ehrlich, and L. H. Miller. 1994. Gender in medical encounters: An analysis of physician and patient communication in a primary care setting. Health Psychology 13:384-92. doi:10.1037/0278-6133.13.5.384.

10. Hall, J. A., and D. L. Roter. 2002. Do patients talk differently to male and female physicians? A meta-analytic review. Patient Education and Counseling 48:217-24. doi:10.1016/S0738-3991(02)00174-X.

11. Hedegaard, J., H. Ahl, A. Rovio-Johansson, and E. Siouta. 2014. Gendered communicative construction of patients in consultation settings. Women \& Health 54:51329. doi:10.1080/03630242.2014.903551. [Taylor \& Francis Online],

12. Heijmans, M., P. Spreeuwenberg, and M. Rijken. 2010. Ontwikkelingen in de zorg voor chronische zieken: Rapportage 2010. Utrecht, Netherlands: NIVEL.

13. Heiligers, P. J. M., J. Noordman, J. C. Korevaar, S. Dorsman, L. Hingstman, A. M. van Dulmen, and D. H. de Bakker. 2012.Praktijkondersteuners in de huisartspraktijk (POH's), klaar voor de toekomst? [Practice nurses in general practice, ready for the future?] Utrecht, Netherlands: NIVEL.

14. Hingstman, L., and R. J. Kenens. 2010. Cijfers uit de registratie van huisartsen. Peiling 2010. Utrecht, Netherlands: NIVEL.

15. Hooper, E. M., L. M. Comstock, J. M. Goodwin, and J. S. Goodwin. 1982. Patient characteristics that influence physician behavior. Medical Care 20:630-38. doi:10.1097/00005650-198206000-00009.

16. Lane, C. 2002. The Behaviour Change Counselling Index (BECCI), Manual for coding behaviour change 
Noordman, J., Dulmen, S. van. The consequences of task delegation for the process of care: female patients seem to benefit more. Women \& Health: 2016, 56(2), 194-207

counselling.http://motivationalinterview.net/library/BECCIManual.pdf (accessed January 18, 2012).

17. Lane, C., M. Huws-Thomas, K. Hood, S. Rollnick, K. Edwards, and M. Robling. 2005. Measuring adaptations of motivational interviewing: The development and validation of the behavior change counseling index (BECCI). Patient Education and Counseling 56:166-73. doi:10.1016/j.pec.2004.01.003.

18. Laurant, M., D. Reeves, R. Hermens, J. Braspenning, R. Grol, and B. Sibbald. 2005. Substitution of doctors by nurses in primary care. Cochrane Database of Systemic Reviews 2: CD001271.

19. Lein, C., and C. E. Wills. 2007. Using patient-centered interviewing skills to manage complex patient encounters in primary care. Journal of the American Academy of Nurse Practitioners 19:215-20. doi:10.1111/jaan.2007.19.issue-5.

20. Meadus, R. J. 2000. Men in nursing: Barriers to recruitment. Nursing Forum 35:5-12. doi:10.1111/nuf.2000.35.issue-3.

21. Meeuwesen, L., C. Schaap, and C. Van der Staak. 1991. Verbal analysis of doctorpatient communication. Social Science \& Medicine 32:1143-50. doi:10.1016/02779536(91)90091-P.

22. Miller, W. R., and S. R. Rollnick. 2002. Motivational interviewing: Preparing people for change, 2nd ed. New York: The Guilford Press.

23. NHG (Nederlands Huisartsen Genootschap). 2012.NHG-

Standaarden.http://nhg.artsennet.nl/kenniscentrum/k_richtlijnen/k_nhgstandaarden.htm (ac cessed January 18, 2012).

24. Noldus, L. P., R. J. Trienes, A. H. Hendriksen, H. Jansen, and R. G. Jansen. 2000. The Observer-Video-Pro: New software for the collection, management and presentation of time-structured data from videotapes and digital media films. Behavior Research Methods, Instruments, \& Computers 32:197-206. doi:10.3758/BF03200802.

25. Noordman, J., E. de Vet, T. van der Weijden, and S. van Dulmen. 2013b. Motivational interviewing within the different stages of change: An analysis of practice nurse-patient consultations aimed at promoting a healthier lifestyle. Social Science \& Medicine 87:60-67. doi:10.1016/j.socscimed.2013.03.019.

26. Noordman, J., B. Koopmans, J. C. Korevaar, T. van der Weijden, and S. van Dulmen. 2013a. Exploring lifestyle counselling in routine primary care consultations: The professionals' role. Family Practice 30:332-40. doi:10.1093/fampra/cms077.

27. Noordman, J., I. van der Lee, M. Nielen, T. Van Der Weijden, and S. Van Dulmen. 2012. Do trained practice nurses apply motivational interviewing techniques in primary care consultations? Journal of Clinical Medicine Research 4:393-401.

28. Noordman, J., P. Verhaak, I. van Beljouw, and S. van Dulmen. 2010. Consulting room computers and their effect on general practitioner-patient communication. Family Practice 27:644-51. doi:10.1093/fampra/cmq058.

29. Rabe-Hesketh, S., A. Skrondal, and A. Pickles. 2002. Reliable estimation of generalized linear mixed models using adaptive quadrature. The Stata Journal 2:1e21.

30. Roter, D. L., and J. A. Hall. 2004. Physician gender and patient-centered communication: A critical review of empirical research. Annual Review of Public Health 25:497-519. doi:10.1146/annurev.publhealth.25.101802.123134.

31. Roter, D. L., J. A. Hall, and Y. Aoki. 2002. Physician gender effects in medical communication. A meta-analytic review. JAMA288:756-64. doi:10.1001/jama.288.6.756.

32. Sim, J., and C. C. Wright. 2005. The kappa statistic in reliability studies: Use, interpretation, and sample size requirements.Physical Therapy 85:257-68.

33. Stewart, M., J. B. Brown, A. Donner, I. R. McWhinney, J. Oates, W. W. Weston, and J. Jordan. 2000. The impact of patient-centered care on outcomes. The Journal of Family Practice 49:796-804.

34. Street, R. L. 2002. Gender differences in healthcare provider-patient communication: Are they due to style, stereotypes, or accommodation? Patient Education and Counseling 48:201-06. doi:10.1016/S0738-3991(02)00171-4.

35. Swenson, S. L., S. Buell, P. Zettler, M. White, D. C. Ruston, and B. Lo. 2004. Patientcentered communication: Do patients really prefer it? Journal of General Internal Medicine 19:1069-79. doi:10.1111/j.1525-1497.2004.30384.x. 
Noordman, J., Dulmen, S. van. The consequences of task delegation for the process of care: female patients seem to benefit more. Women \& Health: 2016, 56(2), 194-207

36. Van den Brink-Muinen, A., S. van Dulmen, V. Messerli-Rohrbach, and J. Bensing. 2002. Do gender-dyads have different communication patterns? A comparative study in WesternEuropean general practices. Patient Education and Counseling48:253-64. doi:10.1016/S0738-3991(02)00178-7.

37. Van Thiel, J., P. Ram, and J. Van Dalen. 2000. Maas-global manual. Limberg, Netherlands: Maastricht University.

38. Waitzkin, H. 1985. Information-giving in medical care. Journal of Health and Social Behavior 26:81-101. doi:10.2307/2136599.

39. Wolosin, R. J., and S. B. Gesell. 2006. Physician gender and primary care patient satisfaction: No evidence of "feminization". Quality Management in Health Care 15:96-103. doi:10.1097/00019514-200604000-00004.

\section{TABLES AND FIGURES}

Table 1. Regression coefficients of practice nurses' application of generic communication skills during consultations with male (Ref) versus female patients.

\begin{tabular}{lccc}
\hline Generic communication skills (Maas-Global) & Regression coefficient $(95 \% \mathrm{Cl})$ & $p$ Value & ICC \\
\hline Introduction $(n=170)$ & $-0.12(-0.88-0.63)$ & .75 & 0.15 \\
Follow-up consultation $(n=153)$ & $-0.21(-0.60-0.19)$ & .31 & 0.20 \\
Request for help $(n=170)$ & $-0.23(-0.87-0.42)$ & .49 & 0.00 \\
Physical examination $(n=159)$ & $0.27(-0.11-0.66)$ & .17 & 0.33 \\
Diagnosis $(n=170)$ & $0.16(-0.20-0.52)$ & .38 & 0.05 \\
Management $(n=169)$ & $-0.29(-0.58--0.01)$ & $.04^{*}$ & 0.23 \\
Evaluation of consultation $(n=170)$ & $-0.05(-0.74-0.65)$ & .90 & 0.13 \\
Exploration $(n=171)$ & $0.02(-0.24-0.27)$ & .90 & 0.19 \\
Emotions $(n=171)$ & $0.54(-0.17-1.24)$ & .14 & 0.12 \\
Information giving $(n=171)$ & $0.29(0.02-0.55)$ & $.03^{*}$ & 0.11 \\
Summarizations $(n=171)$ & $0.38(-0.12-0.87)$ & .14 & 0.15 \\
Structuring $(n=171)$ & $0.01(-0.23-0.25)$ & .93 & 0.32 \\
Empathy $(n=170)$ & $0.13(-0.02-0.27)$ & .09 & 0.01 \\
\hline
\end{tabular}

Note. ICC = intra class correlation on practice nurse level; $\mathrm{Cl}=$ confidence interval. Ten to twenty-eight cases were excluded due to missing data (i.e., not applicable items were coded as missing and missing data on education level of the patient $[n=10]$ ).

*Significant difference of nurses application of communication skill between male (ref) and female patients $(p<.05)$, controlled for patient's education level using multilevel linear regression analysis (except for the items "introduction," "request for help," "evaluation of consultation," and "emotions" for which multilevel logistic regression analysis was used).

Table 2. Regression coefficients of practice nurses' clinical competence during consultations with male (Ref) versus female patients.

\begin{tabular}{lccc}
\hline Clinical competence (adherence to practice guidelines; & & & \\
Maas-Global) & Regression coefficient $(95 \% \mathrm{Cl})$ & $p$ Value & ICC \\
\hline History taking $(n=171)$ & $0.01(-0.34-0.37)$ & .94 & 0.13 \\
Physical examination $(n=160)$ & $-0.07(-0.26-0.11)$ & .42 & 0.22 \\
Diagnosis $(n=170)$ & $-0.15(-0.34-0.03)$ & .10 & 0.08 \\
Management $(n=169)$ & $-0.11(-0.31-0.09)$ & .30 & 0.09 \\
\hline
\end{tabular}

Note. ICC = intra class correlation on practice nurse level; $\mathrm{Cl}=$ confidence interval. Ten to twenty-one cases were excluded due to missing data (i.e., not applicable items of the Maas-Global were coded as missing and missing data on education level of the patient $[n=10]$ ). Results are based on multilevel linear regression analysis, controlled for patient's education level. 
Noordman, J., Dulmen, S. van. The consequences of task delegation for the process of care: female patients seem to benefit more. Women \& Health: 2016, 56(2), 194-207

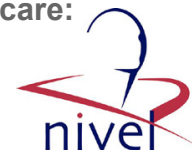

Table 3. Regression coefficients of practice nurses' application of motivational interviewing skills during consultations with male patients (Ref) versus female patients.

\begin{tabular}{llcl}
\hline Motivational interviewing skills (BECCI) & Regression coefficient $(95 \% \mathrm{Cl})$ & $p$ Value & ICC \\
\hline Domains: & & & \\
1. Agenda setting and permission seeking & $0.15(-0.02-0.32)$ & .09 & 0.14 \\
2. The why and how of change in behavior & $0.24(0.04-0.44)$ & $.02^{*}$ & 0.14 \\
3. The whole consultation & $0.34(0.11-0.57)$ & $<.01^{*}$ & 0.14 \\
4. Talk about targets & $0.24(-0.01-0.49)$ & .06 & 0.07 \\
BECCl mean sum score & $0.25(0.08-0.43)$ & $<.01^{*}$ & 0.15 \\
\hline
\end{tabular}

Note. ICC $=$ intra class correlation on practice nurse level; $\mathrm{Cl}=$ confidence interval. Ten cases were excluded due to missing data on education level of the patient ( $n=171$ for all domains).

*Significant difference of nurses application of motivational interviewing skill between male (ref) and female patients $(p<.05)$, controlled for patient's education level using multilevel linear regression analysis.

Table 4. Regression coefficients of lifestyle behavior discussed during consultations with male patients (Ref) versus female patients.

\begin{tabular}{lccc}
\hline Discussing patient's lifestyle behavior & Regression coefficient $(95 \% \mathrm{Cl})$ & $p$ Value & ICC \\
\hline Smoking & $0.09(-0.58-0.75)$ & .79 & 0.07 \\
Alcohol & $-0.16(-0.86-0.54)$ & .66 & 0.04 \\
Physical activity & $0.59(-0.19-1.37)$ & .14 & 0.05 \\
Nutrition & $0.35(-0.43-1.12)$ & .38 & 0.14 \\
\hline
\end{tabular}

Note. ICC = intra class correlation on practice nurse level; $\mathrm{Cl}=$ confidence interval. Ten cases were excluded due to missing data on education level of the patient ( $n=171$ for all lifestyle items). Results are based on multilevel logistic regression analysis, controlled for patient's education level. 